\title{
p-Carbon polarimetry at RHIC
}

\author{
I. Nakagawa ${ }^{*}, \dagger$, I. Alekseev"*, A. Bazilevsky ${ }^{\ddagger}$, A. Bravar ${ }^{\S}$, G.Bunce ${ }^{\ddagger \dagger}$, S. Dhawan

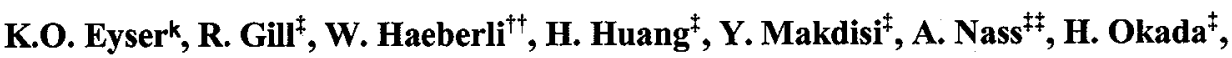 \\ E. Stephenson $^{\S \S}$, D.N. Svirida ${ }^{*-}$, T. Wise ${ }^{\dagger \dagger}$, J. Wood $^{\ddagger}$, A. Zelenski ${ }^{\ddagger}$ \\ -RIKEN, 2-1 Hirosawa Wako, Saitama 351-0198, Japan \\ ${ }^{\dagger}$ RIKEN-BNL Research Center, Upton, NY 11973, USA \\ *Institute for Theoretical and Experimental Physics (ITEP), 117259 Moscow, Russia \\ $\ddagger$ Brookhaven National Laboratory, Upton, NY 11973, USA \\ $\S$ University of Geneva, 1205 Geneva, Switzerland \\ IYale University, New Haven, CT 06520, USA \\ kUniversity of California, Riverside, CA 92521, USA \\ $\dagger$ University of Wisconsin, WI 53706, USA \\ $\$$ $¥$ University of Erlangen, 91058 Erlangen, Germany \\ §§Indiana University Cyclotron Facility, Bloomington, IN 47408, USA
}

Presented at PSTP2007: The International Workshop on Polarized Ion Sources,

Targets, and Polarimetry

Brookhaven National Laboratory, Upton, NY

September 10-14, 2007

March 2008

\section{Collider-Accelerator Department}

\section{Brookhaven National Laboratory \\ P.O. Box 5000 \\ Upton, NY 11973-5000 \\ www.bnl.gov}

\begin{abstract}
Notice: This manuscript has been authored by employees of Brookhaven Science Associates, LLC under Contract No. DE-AC02-98CH10886 with the U.S. Department of Energy. The publisher by accepting the manuscript for publication acknowledges that the United States Government retains a non-exclusive, paid-up, irrevocable, world-wide license to publish or reproduce the published form of this manuscript, or allow others to do so, for United States Government purposes.
\end{abstract}

This preprint is intended for publication in a journal or proceedings. Since changes may be made before publication, it may not be cited or reproduced without the author's permission. 


\section{DISCLAIMER}

This report was prepared as an account of work sponsored by an agency of the United States Government. Neither the United States Government nor any agency thereof, nor any of their employees, nor any of their contractors, subcontractors, or their employees, makes any warranty, express or implied, or assumes any legal liability or responsibility for the accuracy, completeness, or any third party's use or the results of such use of any information, apparatus, product, or process disclosed, or represents that its use would not infringe privately owned rights. Reference herein to any specific commercial product, process, or service by trade name, trademark, manufacturer, or otherwise, does not necessarily constitute or imply its endorsement, recommendation, or favoring by the United States Government or any agency thereof or its contractors or subcontractors. The views and opinions of authors expressed herein do not necessarily state or reflect those of the United States Government or any agency thereof. 


\title{
p-Carbon Polarimetry at RHIC
}

\author{
I. Nakagawa*, ${ }^{*}$, I. Alekseev ${ }^{* *}$, A. Bazilevsky ${ }^{\ddagger}$, A. Bravar ${ }^{\S}$, G. Bunce ${ }^{\ddagger, \dagger}$, \\ S. Dhawan ${ }^{\rrbracket}$, K.O. Eyser"l , R. Gill ${ }^{\ddagger}$, W. Haeberli ${ }^{\dagger \dagger}$, H. Huang ${ }^{\ddagger}$, Y. Makdisi ${ }^{\ddagger}$,

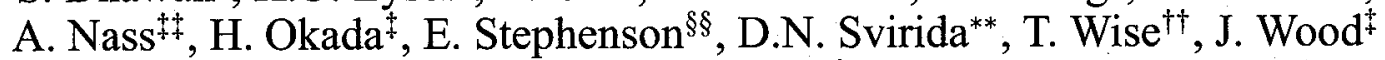 \\ and A. Zelenski ${ }^{\ddagger}$ \\ ${ }^{*}$ RIKEN, 2-1 Hirosawa Wako, Saitama 351-0198, Japan \\ ${ }^{\dagger}$ RIKEN-BNL Research Center, Upton, NY 11973, USA \\ ** Institute for Theoretical and Experimental Physics (ITEP), 117259 Moscow, Russia \\ ${ }^{\ddagger}$ Brookhaven National Laboratory, Upton, NY 11973, USA \\ ${ }^{\S}$ University of Geneva, 1205 Geneva, Switzerland \\ \Yale University, New Haven, CT 06520, USA \\ . University of California, Riverside, CA 92521, USA \\ ${ }^{\dagger \dagger}$ University of Wisconsin, WI 53706, USA \\ $\ddagger$ University of Erlangen, 91058 Erlangen, Germany \\ $\$ \S$ Indiana University Cyclotron Facility, Bloomington, IN 47408, USA
}

\begin{abstract}
The polarization measurement through elastic $(\vec{p}, \mathrm{C})$ reaction plays a crucial role in the polarized proton beam operation of Relativistic Heavy Ion collider at Brookhaven National Laboratory. As well as measuring the polarization, the unknown analyzing power $A_{\mathrm{N}}$ of elastic $(\vec{p}, \mathrm{C})$ is determined as well in combination with the absolute polarization meausement by a $\mathrm{H}$ jet polarimeter. The systematic uncertainty of the Run05 measurements are discussed as well as introducing the experimental apparatus of the polarimeter system.
\end{abstract}

Keywords: polarimeter, $\mathrm{CNI}$, elastic scattering, polarized proton beam

PACS: $13.88 .+\mathrm{e}, 29.27 . \mathrm{Hj}$

\section{INTRODUCTION}

The polarization of the proton beams at the Relativistic Heavy Ion Collider (RHIC) is measured using both a hydrogen jet (H-Jet) $[1,2]$ and carbon polarimeters $[3,5]$. These polarimeters are set up in the 12 o'clock area in the RHIC ring. The H-Jet polarimeter is located at the collision point allowing measurements of both beams. Two identical pC-polarimeters are equipped in the yellow and blue rings, where the rings are separated. The $\mathrm{pC}$-polarimeter measures relative polarization to a few percent statistical accuracy within 20 to 30 seconds using an ultra-thin (typically $10 \sim 20 \mu \mathrm{g} / \mathrm{cm}^{2}$ ) carbon ribbon target, providing fast feedback to beam operations and experiments. The absolute normalization is provided by the H-Jet polarimeter, which measures over $1 \sim 2$ days to obtain $\sim 5 \%$ statistical uncertainty (in Run05). Thus, the operation of the carbon polarimeters was focused on better control of relative stability between one measurement to another measurement rather than measuring the absolute polarization.

The published data of the analyzing power for the elastic polarized proton-carbon scattering is available up to the $21.7 \mathrm{GeV} / c[6]$. There are no published data available at the storage (flat-top) proton beam energy of $100 \mathrm{GeV}$ where the colliding experiment was performed in RHIC. Shown in the Fig. 1 is the analyzing power measured 
by the blue carbon polarimeter during Run04 operation for the extended range of the momentum transfers $-t$. The absolute scale was determined by normalizing the average polarization observed by the carbon polarimeter against the absolute polarization measurements by the hydrogen polarimeter. Nevertheless the precision of the normalization was limited by the statistical accuracy of the jet measurement; $\Delta A_{\mathrm{N}}^{\text {Run04 }} \sim \pm 9$ $\%$. The strategy is to improve the accuracy year by year with more statistical abundance in the average polarization measurements by the carbon $\overline{P_{\overrightarrow{\mathrm{p} C}}^{\mathrm{Run} 05}}$ and the hydrogen $\overline{P_{\overrightarrow{\mathrm{p} p}}^{\text {Run05 }}}$ polarimeters. The improved analyzing power of Run $05 A_{\mathrm{N}}^{\mathrm{Run} 05}$ is given by

$$
A_{\mathrm{N}}^{\mathrm{Run} 05}=A_{\mathrm{N}}^{\mathrm{Run} 04} \frac{\overline{P_{\overrightarrow{\mathrm{pC}}}^{\mathrm{Run} 05}}}{\overline{P_{\overrightarrow{\mathrm{p} p}}^{\mathrm{Run} 05}}} .
$$

The curves in the Fig. 1 are the model predictions[7] of with (blue) and without (red) the spin-flip amplitude fitted to the data. The analyzing power for the elastic polarized proton-carbon scattering is predicted to be maximized at the momentum transfer of $\left(-t \sim 0.003(\mathrm{GeV} / \mathrm{c})^{2}\right)$ due to the interference between the electromagnetic and the strong amplitudes (this is known as the Coulomb-Nuclear Interference (CNI) region). In order to take advantage of relatively large sensitivity to the polarization, the recoil carbon atoms were detected near 90 degrees with respect to the beam direction. Kinetic energy range was from 400 to $900 \mathrm{keV}$, corresponding to a momentum transfer of $0.09<-t<0.23(\mathrm{GeV} / \mathrm{c})^{2}$. The lower the kinetic energy, the larger the analyzing power and the more sensitivity we gain. However, in reality, the present range is constrained by the reliability of the low energy carbon detection as discussed in references $[4,5]$. Since there is a $t$-dependence in the analyzing power even within the limited $t$ coverage, absolute energy of recoil carbon ion needs to be measured to define the kinematics.

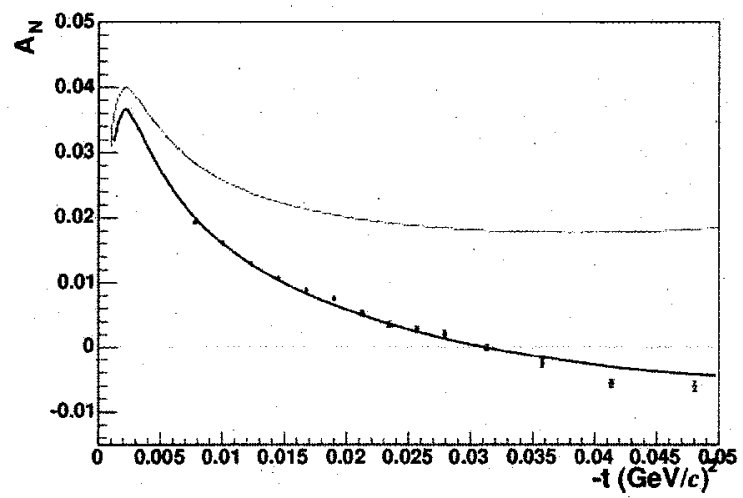

FIGURE 1. The analyzing power measured by the blue carbon polarimeter during Run04 for the extended range of the momentum transfers $-t$. The absolute scale was normalized against the hydrogen polarimeter results in Run04. 


\section{EXPERIMENTAL APPARATUS}

The carbon polarimeters consisted of a carbon target and six silicon strip detectors. They are all mounted in the vacuum inside a scattering chamber as seen in (left panel) Fig. 2. The photograph shows the scattering chambers of the blue and yellow polarimeters mounted on the blue and yellow ring beam pipes, respectively.
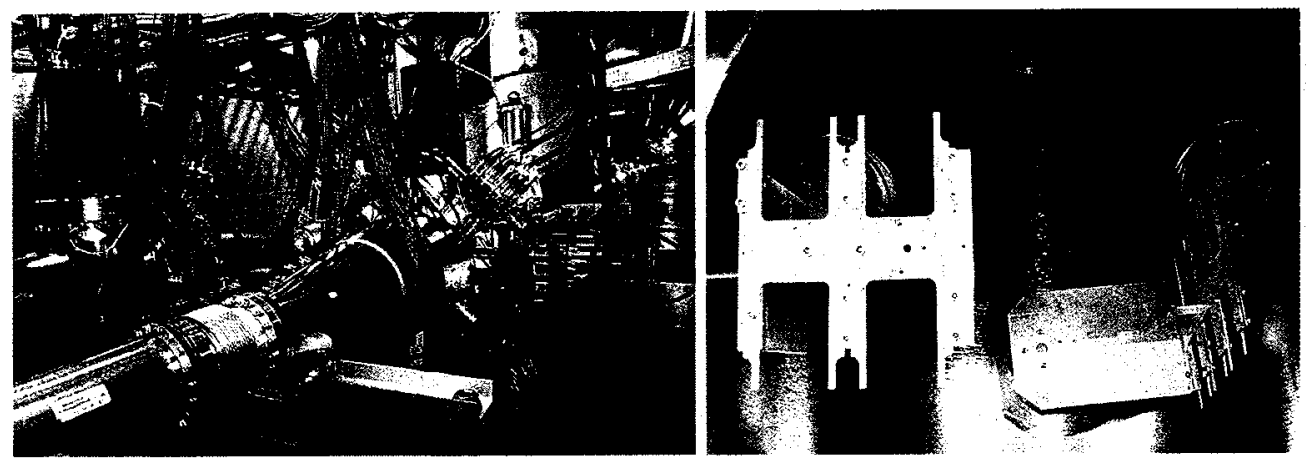

FIGURE 2. (Left) The horizontal and vertical target folders viewed from the top of the scattering chamber. The beam goes from the bottom to the top of the picture. (right) The photograph shows the scattering chambers of the blue and yellow polarimeters mounted on the blue and yellow ring beam pipes, respectively.

Very thin carbon ribbon targets have been developed at Indiana University Cyclotron Facility [8]. The targets were made by vacuum evaporation-condensation onto smooth glass substrates. Typically size of $2.5 \mathrm{~cm}$ length with $10-20 \mu \mathrm{g} / \mathrm{cm}^{2}$ thick and $4 \sim$. $10 \mu \mathrm{m}$ width target was glued both edge on an open side of the "C"-shaped target folder frame as shown in (right panel) Fig. 2. The targets are normally kept away from the beam line and it rotated into the beam only when the polarization measurement is executed, with a choice of 4 vertical and horizontal targets for Run05. The folder with 6 frames in the Fig. 2 is the upgraded version of the vertical target folder for Run08. It is crucial to mount multiple targets simultaneously because the target is so thin, and has a certain lifetime against the radiation damage. The target lasted within a week on average during Run05 and the pre-mounted spare target was used without breaking the vacuum to replace the broken one.

Six silicon sensors were mounted in a vacuum chamber at 45, 90, 135 degrees azimuthally in both left and right sides with respect to the beam with schematic shown in Fig. 3. The sensor has $10 \times 24 \mathrm{~mm}^{2}$ total active area, divided into 12 strips of $10 \times 2$ $\mathrm{mm}^{2}$ each as shown in the left panel of Fig. 4. The segmented axis of the detectors are oriented to the azimuthal direction, so there is no segmentation of the detectors in the beam direction. Thus the present setup do not have any sensitivity to the scattering angle of the recoil carbon ions within the acceptance. The thickness of the detector is $400 \mu \mathrm{m}$, fully depleted with the operation bias voltage of 100 to $150 \mathrm{~V}$. The strips are made by the Boron implantation p+-doping to a depth of $150 \mathrm{~nm}$ on the n-type Si bulk on the 
side facing the target. ${ }^{1}$ The middle panel in Fig. 4 illustrates the cross section of the silicon sensor. The detectors were mounted on the one end of the detector holder whose flange on the other end was mounted on the scattering chamber maintaining distance from target to the silicon sensors to be $18.5 \mathrm{~cm}$.

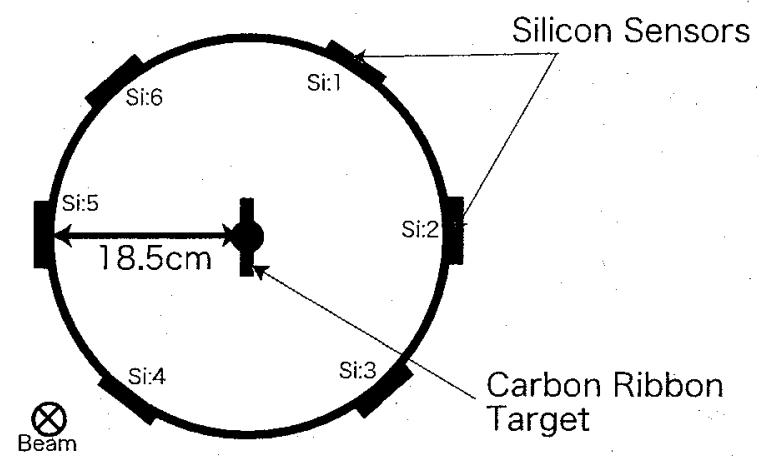

FIGURE 3. The RHIC pC-polarimeter setup. Silicon sensors are aligned 45, 90, and 135 degrees azimuthally in both left and right side with respect to the beam direction. The beam is pointing into the figure perpendicularly.
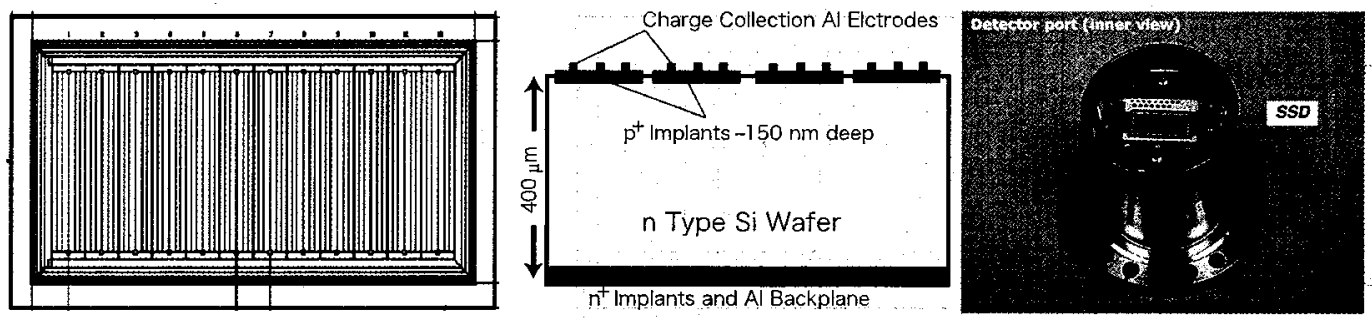

FIGURE 4. (Left) the mechanical drawing of the silicon sensor. Each sensor is segmented into 12 strips with $2 \mathrm{~mm}$ pitch. (Middle) The cross section of the silicon sensor. (Right)The silicon detector mounted on a support structure attached on a flange.

\section{EVENT RECONSTRUCTION AND EVENT SELECTION}

As it is described in the introduction, extending the energy range to lower region is certainly an advantage in terms of 1) the larger magnitude of $A_{\mathrm{N}}$ in lower $-t$ and 2) larger cross section (more statistics). However, the practical range is limited by the tolerable size of the uncertainty to reconstruct the energy in the low energy region. The current $d E / d x$ model to describe the energy loss in a "effective" dead-layer ${ }^{2}$ fits very well in the region $E \geq 500 \mathrm{keV}$ (residual $\sim 0$ ), while it tends to undershoot the data $E<500 \mathrm{keV}$

\footnotetext{
1 The layer to the depth of the Boron implantation is so called dead-layer and corresponding thickness is $150 \mathrm{~nm} \times 2.33 \mathrm{~g} / \mathrm{cm}^{3} \approx 35 \mu \mathrm{g} / \mathrm{cm}^{2}$, which is reasonably consistent with what were initially observed in the both blue and yellow polarimeters at the beginning of Run 05

${ }^{2}$ The effective dead-layer is the dead-layer plus inefficient charge collection region around the surface of the silicon detector. The energy correction for the energy loss in this region is discussed in references $[4,5]$
} 
(residual $<0$ ). Typically the residual gets about $-0.5 \mathrm{~ns}$ at $E=400 \mathrm{keV}$ and rare, but worst case, $-1 \mathrm{~ns}$ at $E=900 \mathrm{keV}$. Such a systematic tendency suggests an inconsistency between current $d E / d x$ model with data. Thus we set the lower energy limit to be 400 $\mathrm{keV}$ whose corresponding error is studied below.

The error of $\pm 1 \mathrm{~ns}$ in $t_{0}$ determination can be calculated by taking the derivative of kinetic energy formula by $t$ :

$$
E=\frac{1}{2} M \frac{L^{2}}{t_{\mathrm{tof}}^{2} c^{2}} \frac{\partial E}{\partial t_{\mathrm{tof}}}=-M \frac{L^{2}}{t_{\mathrm{tof}}^{3} c^{2}}=-\frac{2 \sqrt{2} c}{L \sqrt{M}} E \sqrt{E}
$$

The effects are about $11 \mathrm{keV}$ at $E=400 \mathrm{keV}$ and $37 \mathrm{keV}$ at $E=900 \mathrm{keV}$ (approximately corresponding effective dead-layer is $4 \mu \mathrm{g} / \mathrm{cm}^{2}$ ). Thus overall uncertainty due to the precision of $t_{0}$ determination by $\pm 1 \mathrm{~ns}$ results in the energy determination of $3 \sim 4 \%$ within the energy range $400 \leq E \leq 900 \mathrm{keV}$.

\section{Invariant Mass Cut}

Shown in the Fig. 5 is the typical time-of-flight and the kinetic energy plot reconstructed using the best fit parameters of the dead-layer fit $[4,5]$. Dotted and solid curves show the 2 and $3 \sigma$ cut of the invariant mass as shown in the left panel in Fig.6. The small peak seen below $4 \mathrm{GeV}$ consisted of $\alpha$ backgrounds whose peak does not necessarily appear at the right $\alpha$ mass because the energy loss in the effective dead-layer was calculated assuming the carbon ion mass. A contamination of the $\alpha$ background underneath the carbon invariant mass peak is typicaly less than $1 \%$ within $3 \sigma$ from the nominal carbon mass position. Within $3 \sigma$ cuts, the number of the elastic carbons are observed about $200 \sim 300$ thousand events. About $50 \%$ of accumulated events from raw data were dropped after the energy and the $3 \sigma$ cuts were applied.

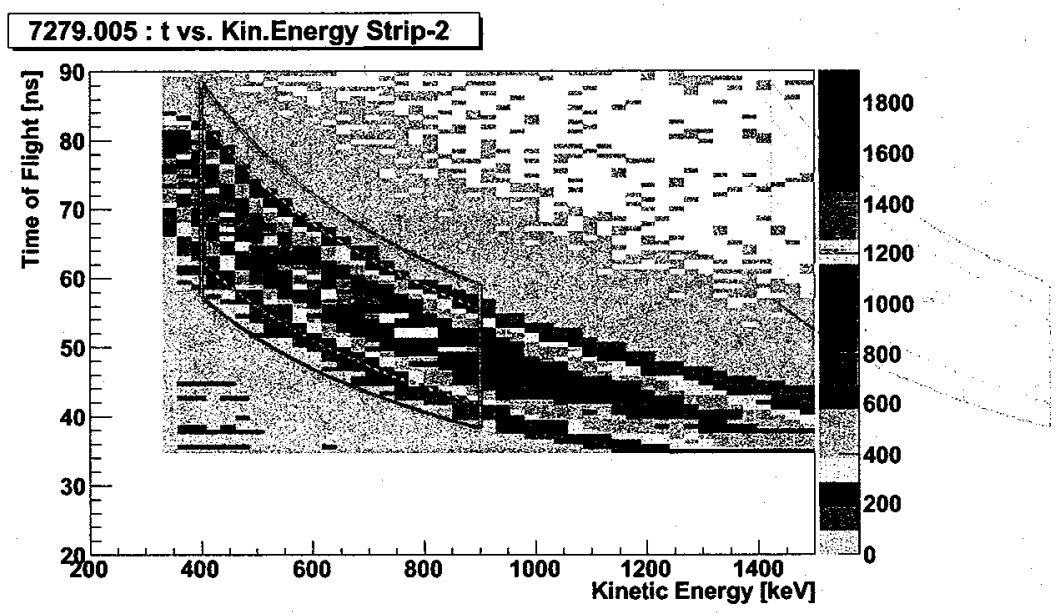

FIGURE 5. Time of flight and reconstructed kinetic energy correlation plot after the energy correction. Dotted and solid curves show 2 and $3 \sigma$ from carbon mass in the invariant mass distribution. 

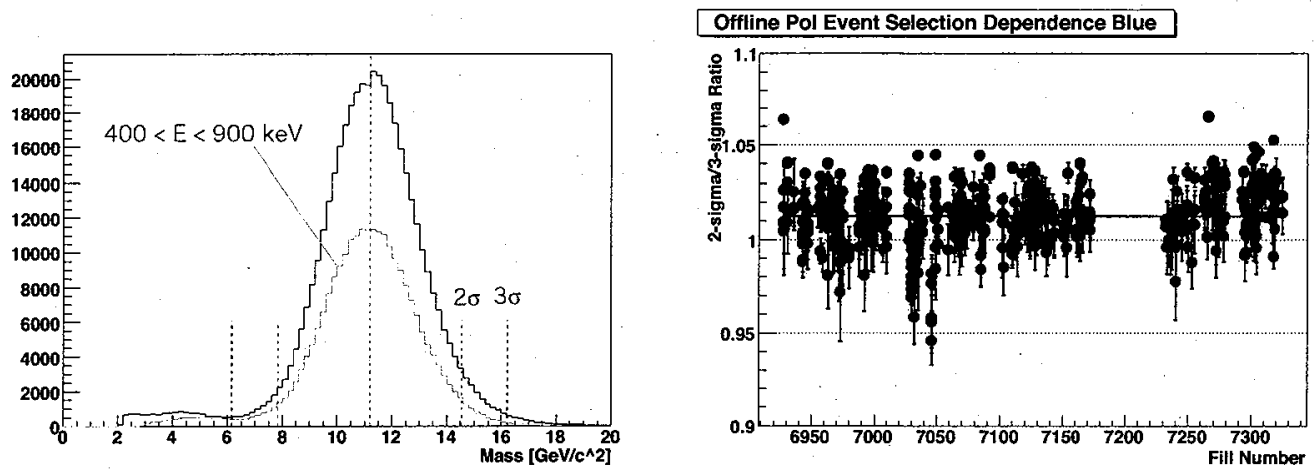

FIGURE 6. (Left) A typical invariant mass distribution. The red histogram shows the invariant mass for the events $400<E<900 \mathrm{keV}$. Dotted and solid curves represents 2 and $3 \sigma$ from carbon mass. (Right) The ratio of the polarizations calculated by $2 \sigma$ and $3 \sigma$ invariant mass cuts for blue polarimeters. The horizontal bar shows the average.

The effect of the backgrounds contamination underneath the carbon invariant mass was studied by comparing the polarizations between $2 \sigma$ and $3 \sigma$ invariant mass cuts. Shown in right panel of Fig. 6 are ratio of the polarizations calculated by $2 \sigma$ and $3 \sigma$ invariant mass cuts for blue (left) and yellow (right) polarimeters. As can be seen in the figure, the polarizations derived by the $2 \sigma$ cut are about $1 \%$ higher than the $3 \sigma$ cut. This result suggests the more background contamination in $3 \sigma$ cut case drives the lower polarization.

The exterpolation of the polarization at the $0 \sigma$ cut is necessary in order to evaluate the polarization under the circumstances of zero background contamination. However, this is not necessary in our case because this effect is absorbed into the "effective" analyzing power of proton-Carbon as a consequence of the normalization against the H-Jet average polarization. This is valid as long as we keep the $\sigma$ cut of the invariant mass consistent with data go to H-Jet normalization and polarizations provided to experiments. In other word, the analyzing power determined by the normalization is "optimized" to make the average polarization measured by the proton-Carbon polarimeter to be consistent with the absolute polarization measurement by the H-Jet polarimeter. Thus we do not assign error from the background contamination to the polarizations measured by the protonCarbon polarimeters.

\section{RUN BY RUN POLARIZATION}

AVERAGE $A_{\mathrm{N}}$ : The carbon events which passed kinematic cuts $(|\Delta M| \leq 3 \sigma$ and $400 \leq$ $E \leq 900 \mathrm{keV}$ ) were then integrated over the energy range. As it is discussed later, the polarization is extracted through the strip by strip asymmetries cacluclated using the selected events per strip. The observed asymmetries were devided by the average $\overline{A_{\mathrm{N}}}$ to convert the asymmetry into the polarization. The $\overline{A_{\mathrm{N}}}$ is the average analyzing power within the energy range of the event selection. It is calculated by averaging $A_{\mathrm{N}}\left(E_{i}\right)$ weighted by the yields $Y[i]$ of the $i$ th bin in the energy spectrum. $E_{i}$ is the $i$ th bin of 
the energy $E$.

$$
\overline{A_{\mathrm{N}}}=\frac{\sum_{i}^{N} A_{\mathrm{N}}\left(E_{i}\right) \times Y[i]}{\sum_{i}^{N} Y[i]}
$$

where $N$ is the total number of bins in the energy spectrum. In RUN05 analysis the the energy spectrum histogram range from zero to $1500 \mathrm{keV}$ was binned by 180 . The number of bins are 59 between the energy range $400 \leq E \leq 900 \mathrm{keV}$. $i$ runs for the maximum bins up to 1500 though, bins out side $400 \leq \bar{E} \leq 900 \mathrm{keV}$ obviously do not contribute on $\overline{A_{\mathrm{N}}}$ because $Y[i]=0$.

Shown in Fig. 7 is the typical energy spectrum of the carbon events combined for all active strips after the kinematic cuts. The curve is a model prediction[7] of $A_{\mathrm{N}}(E)$ scaled by the RUN04 data.



FIGURE 7. The typical energy spectrum of the carbon events combined all active strips after the kinematic cuts. The curve is a model prediction of $A_{\mathrm{N}}(E)$ scaled by the RUN04 data. Dashed line shows the weighted average $\overline{A_{\mathrm{N}}}$.

$\operatorname{SIN}(\phi)$ FIT: The run-by-run polarization is calculated based on the strip asymmetries, combining all bunch-by-bunch asymmetries. The asymmetry of strip $i$ is calculated using the number of elastic carbon events after the kinematic cuts for all positive bunches $N_{i}^{+}$ and negative bunches $N_{i}^{-}$in strip $i$ :

$$
A_{i}=\frac{N_{i}^{+}-R_{i} N_{i}^{-}}{N_{i}^{+}+R_{i} N_{i}^{-}}, \quad R_{i}=\frac{\sum_{j \neq i, 37-i, 36+i, 72-i}^{72} N_{j}^{+}}{\sum_{j \neq i, 37-i, 36+i, 72-i}^{72} N_{j}^{-}}
$$

where $i$ runs for active strips up to 72 and $R_{i}$ is the luminosity ratio for the strip $i$. In order to remove the bias effect from the strip $i$ for the luminosity calculation, the strip $i$ is excluded from the luminosity calculation. Also to avoid introducing false asymmetry comes from the geometrical acceptance effect by doing so, not only the strip locates diagonally opposite location, but also ones located at cross geometries are excluded as well (total 4 strips).

Plotted in Fig. 8 with solid circles are typical examples of so calculated strip asymmetries divided by the $\overline{A_{\mathrm{N}}}$ as a function of the azimuthal angle in the unit of radian. 
The observed polarization for detectors in D1, D3, D4, D6 are suppressed due to the sensitivity to the vertical polarization by $\sqrt{2}$ compared to the detectors D2 and D3.

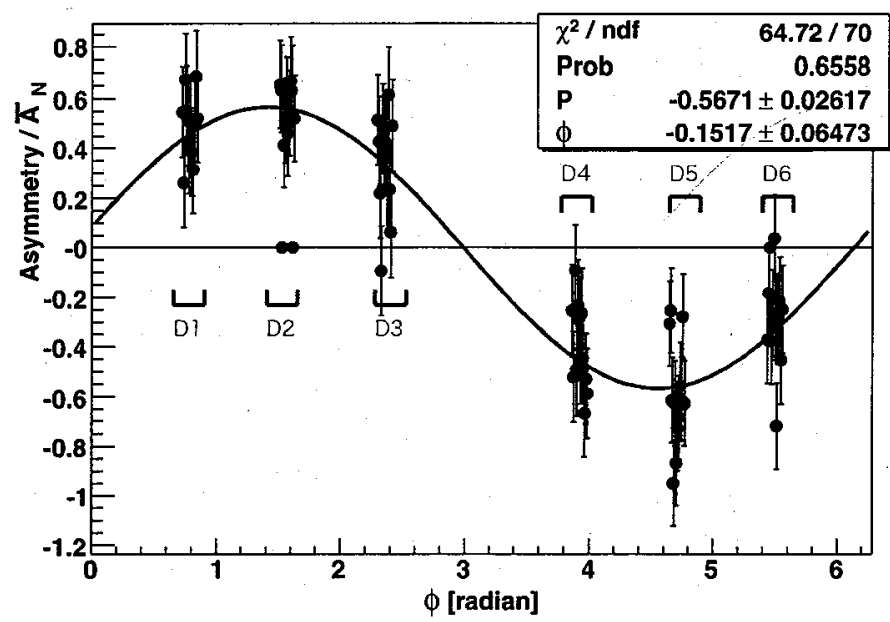

FIGURE 8. The strip by strip polarization plotted as a function of the azimuthal angle (rad). The red curve represents the best fit to the data of function 5 .

The strip by strip polarizations are then fitted with the sin function

$$
P(\phi)=P \sin (\phi+\Delta \phi)
$$

where $P$ is strip averaged polarization and $\Delta \phi$ is the radial polarization vector, respectively and they were set as free parameters. The best fit result is drawn by the red curve in the figure. After disabling strips for the suspiciously behaving periods through the QA analysis as discussed previously, the $\chi^{2}$ of the fit distributes around 1 .

\section{AVERAGE POLARIZATION FILL BY FILL}

To normalize with the jet, we decided to obtain averages of the measurements in a fill to obtain a polarization from the $\mathrm{pC}$ polarimeter for each fill. This was done by a $\left(1 / \Delta P_{i}\right)^{2}$, beam-intensity, and time-weighted average of the measurements in a given fill $j$

$$
\bar{P}_{\mathrm{j}}=\frac{\sum_{i} \frac{P_{\mathrm{i}}}{\Delta P_{\mathrm{i}}^{2}} \cdot I_{\mathrm{i}} \cdot \Delta t_{\mathrm{i}}}{\sum_{i} \frac{1}{\Delta P_{\mathrm{i}}^{2}} \cdot I_{\mathrm{i}} \cdot \Delta t_{\mathrm{i}}}
$$

where $i$ runs for "good" measurements in fill $j$ and $\Delta t_{\mathrm{i}}$ represents time interval between the " $i$ "th measurement and " $i+1$ "th measurement to assign more (less) weight to the " $i$ "th polarization measurement if the time interval is long (short) before the next measurement is executed in order to represent the polarization of the particular interval of the fill. The beam intensity $I_{i}$ is calculated by taking sum of the wall current monitor readouts for the active fills (disabled fills were excluded). The wall current monitor readouts are acquired in the $\mathrm{pC}$-polarimeter data stream at the beginning of every data 
taking. The uncertainty $\Delta P_{\mathrm{i}}$ used for each measurement was the statistical uncertainty $\Delta P_{i}^{\text {sta }}$ added quadratically to the polarization profile[9] uncertainty $\Delta P_{i}^{\text {prof }}$.

$$
\Delta P_{\mathrm{i}}^{2}=\sqrt{\left(\Delta P_{\mathrm{i}}^{\text {sta }}\right)^{2}+\left(\Delta P_{\mathrm{i}}^{\text {prof }}\right)^{2}}
$$

For blue, no profile uncertainty; for yellow, an uncertainty depending on the normalized polarimeter rate. The time weighting was used to average over a fill by assigning a weight for each measurement of the time duration polarization up to the midpoint in time until the next measurement. Thus the measurements with significantly lower rate than the expected rate contribute less to the average polarization of that fill.

The uncertainty for the fill polarization is a quadratic sum of the statistical uncertainty from the above approach to obtain the average fill polarization, the contribution from observed fluctuations in the energy correction which affects the polarization $(1.5 \%$ in blue and $1.7 \%$ in yellow, in $\Delta P / P$ ), an uncertainty due to polarization profile[9] $(4.3 \%$ for blue and $5.7 \%$ for yellow in $\Delta P / P)$, and an uncertainty that depends on the number of measurements in the fill that were taken away from the beam center. For the latter, blue had no uncertainty for this (no observed polarization profile) and the yellow uncertainty was taken as the difference in fill polarization correcting for these off-center measurements vs. not correcting for them (note: we do not correct the polarizations; this is a method to obtain the uncertainty only). In this way, fills with off-center measurements have larger uncertainties.

\section{NORMALIZATION}

As a preparation to compare with the polarization measured by the H-Jet polarimeter, the data sets are classified into two groups which are separated by the operation mode of the RHIC beam. The first one is the group of measurements which were done during 56 bunches operation and the second one is these of 112 bunches operation. Both the proton-Carbon and the H-Jet polarimeters were operated in slightly different conditions for these two different RHIC beam operations. Thus this grouping is aimed to check any systematic discrepancies in the agreement between two polarimeters depending on the operation modes.

The average polarizations for two distinct jet measurement periods are then calculated using fill by fill polarization averages $\left(\overline{P_{\mathbf{j}}}\right)$. The average $\overline{P_{k}}$ are weighted by the duration of the jet operation of each fill $\Delta t_{j}^{\text {jet }}$. If the jet operated for only a small fraction of the fill duration, then the $\overline{P_{k}}$ of the fill will contribute less to the average polarization of the given jet measurement period.

$$
\overline{P_{k}}=\frac{\sum_{j} \frac{\overline{P_{j}}}{\sigma_{\mathrm{j}}^{2}} \cdot \Delta t_{\mathrm{j}}^{\mathrm{jet}}}{\sum_{j} \frac{1}{\sigma_{\mathrm{j}}^{2}} \cdot \Delta t_{\mathrm{j}}^{\mathrm{jet}}}
$$

where $j$ runs for fills classified for jet running period $k$.

With the estimate of the polarization measured by the vertical target at the center of the beam for a jet measurement period, we then needed to obtain an intensity-weighted 
average for the polarization, averaged over the horizontal distribution of the beam, in order to compare with the jet measurement. This requires the horizontal polarization profile. Because the vertical target automatically takes an intensity-weighted average over any vertical polarization profile, the required average is only for the horizontal dimension. They are estimated $0.5 \%$ for blue and $2.2 \%$ for yellow [9], in $\Delta A_{N} / A_{N}$. The agreement between the 2004[3] and 2005 jet calibrations is good as shown in Fig. 9. The results are presented as the new analyzing power and uncertainties for the $\mathrm{pC}$ measurements, separately for blue and yellow.

$$
\begin{aligned}
A_{N}^{\text {blue2005 }} & =A_{N}^{2004} \times\left\{1.01 \pm 0.031^{\mathrm{a})} \pm 0.029^{\mathrm{b})} \pm 0.005^{\mathrm{c})}\right\} \\
A_{N}^{\text {yellow2005 }} & =A_{N}^{2004} \times\left\{1.02 \pm 0.028^{\mathrm{a})} \pm 0.029^{\mathrm{b})} \pm 0.022^{\mathrm{c})}\right\}
\end{aligned}
$$

where a) statistical uncertainty from the jet measurement (independent for blue and yellow) b) systematic uncertainty for jet measurement (correlated for blue and yellow) c) systematic uncertainty from horizontal profile uncertainty, independent for blue and yellow.
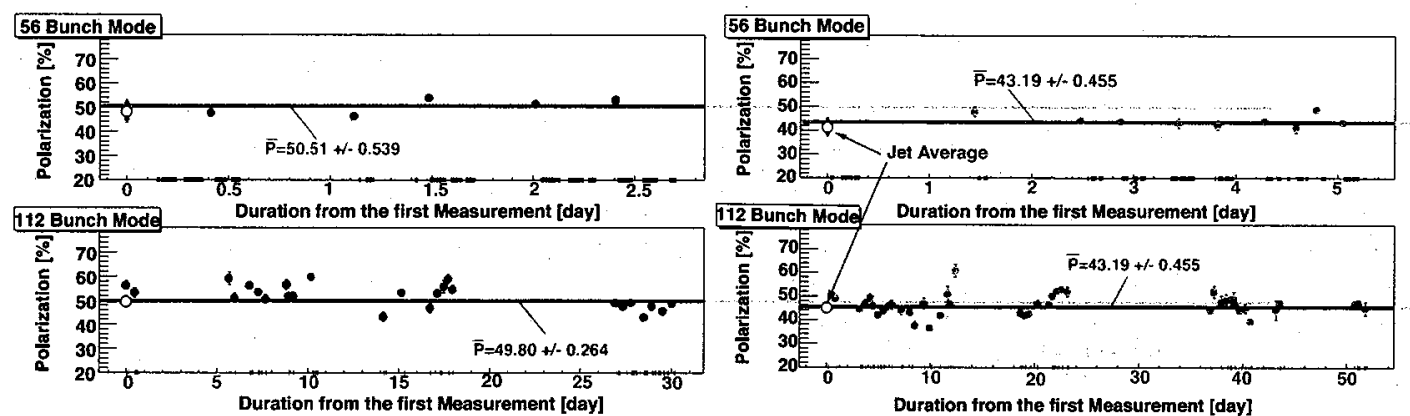

FIGURE 9. Comparison of the average polarizations of the H-jet and blue (left) and yellow (right) proton-Carbon polarimeters. The holizontal lines represent the average of fill-by-fill averaged polarizations of $\mathrm{pC}$ polarimeters (solid circle), whereas open circles represent the average polarization measured by $\mathrm{H}$-jet polarimeter with 56 (top) and 112 bunch (bottom) modes.

\section{REFERENCES}

1. H. Okada et al. Phys. Lett. B638, 450 (2006)

2. K.O. Eyser et al., RHIC/CAD Accelerator Physics Note Vol.274 (2007)

3. O. Jinnouchi et al., RHIC/CAD Accelerator Physics Note Vol.171 (2004)

4. I. Nakagawa et al., $17^{\text {th }}$ International Spin Physics Symposium SPIN2006

5. I. Nakagawa et al., RHIC/CAD Accelerator Physics Note Vol.275 (2007)

6. J. Tojo et al., Phys. Rev. Lett. 89, 052302 (2002).

7. L. Trueman, hep-ph/0305085 (2003).

8. W.R. Lozowski and J.D. Hudson, Nucl. Inst. Meth. A303, 34 (1991), ibid Nucl. Instr. Meth. A334, 173 (1993).

9. A. Bazilevsky, et al., these proceedings 